\title{
Diabetic ketoacidosis producing extreme hyperkalemia in a patient with type 1 diabetes on hemodialysis
}

\author{
Hodaka Yamada', Shunsuke Funazaki', Masafumi Kakei', Kazuo Hara' and \\ San-e Ishikawa²
}

'Division of Endocrinology and Metabolism, Jichi Medical University Saitama Medical Center, Saitama, Japan and 2Division of Endocrinology and Metabolism, International University of Health and Welfare Hospital, Nasushiobara, Japan
Correspondence

should be addressed

to H Yamada

Email

hyamada0510@jichi.ac.jp

\section{Summary}

Diabetic ketoacidosis (DKA) is a critical complication of type 1 diabetes associated with water and electrolyte disorders. Here, we report a case of DKA with extreme hyperkalemia $(9.0 \mathrm{mEq} / \mathrm{L})$ in a patient with type 1 diabetes on hemodialysis. He had a left frontal cerebral infarction resulting in inability to manage his continuous subcutaneous insulin infusion pump. Electrocardiography showed typical changes of hyperkalemia, including absent $\mathrm{P}$ waves, prolonged QRS interval and tented T waves. There was no evidence of total body water deficit. After starting insulin and rapid hemodialysis, the serum potassium level was normalized. Although DKA may present with hypokalemia, rapid hemodialysis may be necessary to resolve severe hyperkalemia in a patient with renal failure.

\section{Learning points:}

- Patients with type 1 diabetes on hemodialysis may develop ketoacidosis because of discontinuation of insulin treatment.

- Patients on hemodialysis who develop ketoacidosis may have hyperkalemia because of anuria.

- Absolute insulin deficit alters potassium distribution between the intracellular and extracellular space, and anuria abolishes urinary excretion of potassium.

- Rapid hemodialysis along with intensive insulin therapy can improve hyperkalemia, while fluid infusions may worsen heart failure in patients with ketoacidosis who routinely require hemodialysis.

\section{Background}

Diabetic ketoacidosis (DKA) is a very common endocrinology emergency. It is usually associated with severe circulatory volume depletion. Management of fluids, metabolic acidosis and electrolyte disorders is mandatory. In DKA, mild-to-moderate elevation of serum potassium is usually seen despite total body potassium wasting (1). After intravenous insulin infusion to treat DKA, even if the initial serum potassium levels are normal or mildly elevated $(5-5.2 \mathrm{mEq} / \mathrm{L})$, potassium replacement is required to prevent insulin therapy-induced hypokalemia (1). Insulin deficiency can alter potassium distribution between the intra- and extracellular spaces. However, patients on hemodialysis because of end-stage renal disease with anuria do not have severe volume depletion 
or renal electrolyte wasting even if they develop DKA. We describe a patient with chronic hemodialysis who had DKA with extreme hyperkalemia.

\section{Case presentation}

A 41-year-old Japanese man with type 1 diabetes had been on chronic hemodialysis (three times per week) due to diabetic nephropathy since the age of 32 years. Dialysis clinic staff called him because he did not go to regular hemodialysis at $10: 00 \mathrm{~h}$ on the day. Then, his family discovered that the patient had disturbed consciousness in his bedroom and called for an ambulance; he was taken to the Jichi Medical University Saitama Medical Center. He had been diagnosed with type 1 diabetes mellitus at the age of 18 years and had been treated with continuous subcutaneous insulin infusion (CSII) pump for the preceding recent one year. He was trained and informed the risk of DKA at the induction of CSII, but he did not have stripes for measuring ketones. The physical findings upon hospitalization included a height of $170 \mathrm{~cm}$ and weight of $59 \mathrm{~kg}$, with a body mass index of 20.4. His normal dry weight was $56.3 \mathrm{~kg}$. His level of consciousness was E3V4M5 (Glasgow Coma Scale). His blood pressure was $70 / 50 \mathrm{mmHg}$, and his pulse rate was regular at 60 beats/min. His skin turgor was not reduced and the mouth was moist. There was an ejection murmur at the apex and bilateral pretibial pitting edema. The neurologic examination revealed right hemiplegia and higher cortical dysfunction (aphasia and acalculia). It was unclear when the neurological symptoms exactly appeared.

\section{Investigation}

The plasma glucose was $1498 \mathrm{mg} / \mathrm{dL}$, serum sodium $106 \mathrm{mEq} / \mathrm{L}$ and potassium $9.0 \mathrm{mEq} / \mathrm{L}$. Arterial blood gas analysis showed a pH of $6.99, \mathrm{HCO}_{3}-$ of $8.9 \mathrm{mmol} / \mathrm{L}$ and base excess of $-21.5 \mathrm{mmol} / \mathrm{L}$. Initial laboratory findings are shown in Table 1 . We did not measure plasma osmolality. Chest x-ray showed cardiac enlargement, and electrocardiography (ECG) revealed typical features associated with hyperkalemia, including absent $\mathrm{P}$ waves, prolonged QRS intervals and tented $\mathrm{T}$ waves (Fig. 1). These physical and laboratory findings suggested DKA with marked hyperkalemia but no evidence of related total body fluid loss. It was thought that his body weight was increased due to the skip of hemodialysis, because he could not go to hemodialysis clinic.
Table 1 Laboratory data on admission.

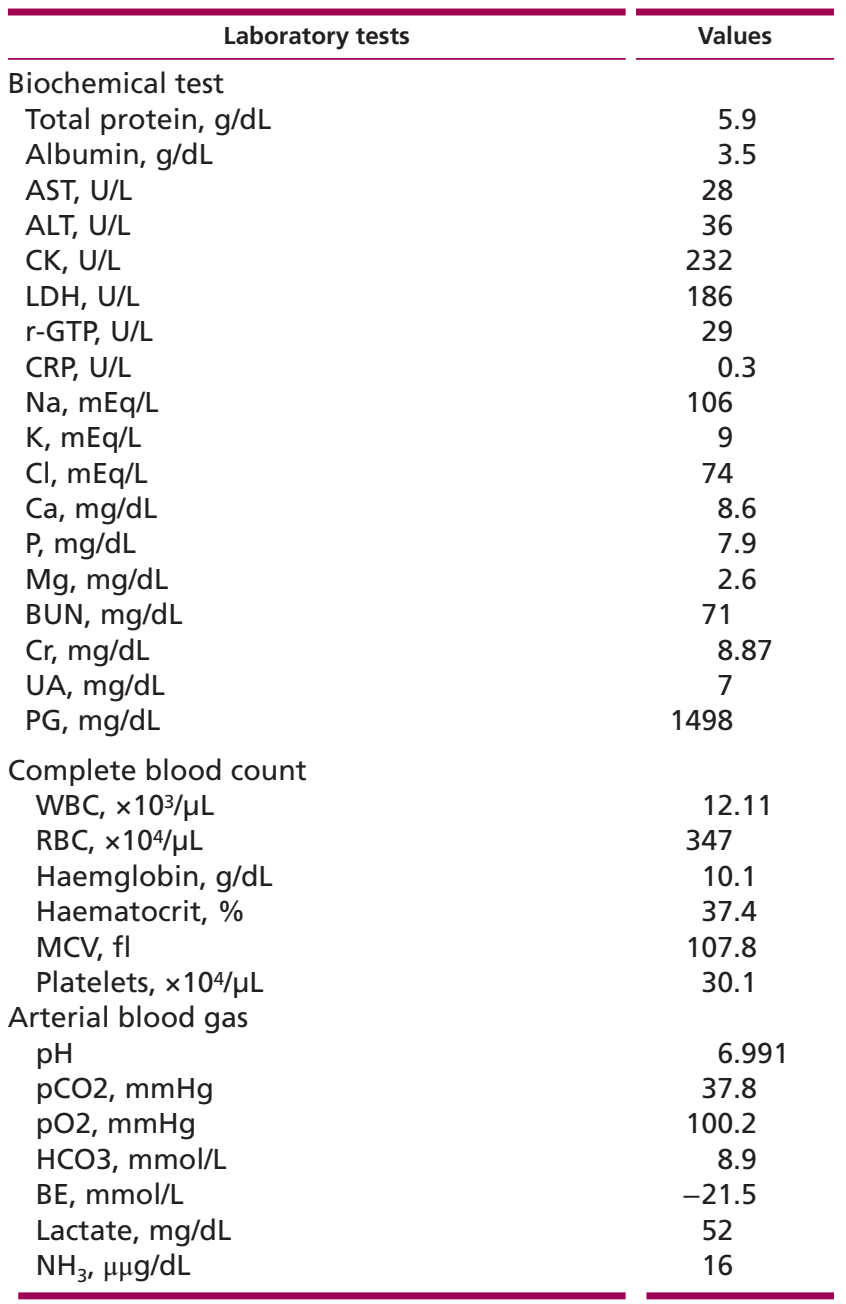

$A B G$; arterial blood gas.

\section{Treatment}

We started an intravenous insulin infusion and hemodialysis to reduce the glucose and potassium levels. Fluid infusions were not given because there was no evidence of a fluid deficit. Four hours after beginning treatment, the plasma glucose was gradually reduced from 1498 to around $400 \mathrm{mg} / \mathrm{dL}$, and serum potassium decreased from 9.0 to $4.0 \mathrm{mEq} / \mathrm{L}$. The hyperkalemic changes disappeared from ECG. On hospital day 2, however, his right hemiplegia persisted. Head computed tomography demonstrated a lowdensity area in the left frontal lobe, indicating a left frontal cerebral infarction. After glycemic control, serum sodium level normalized to baseline level (135$140 \mathrm{mEq} / \mathrm{L})$. 


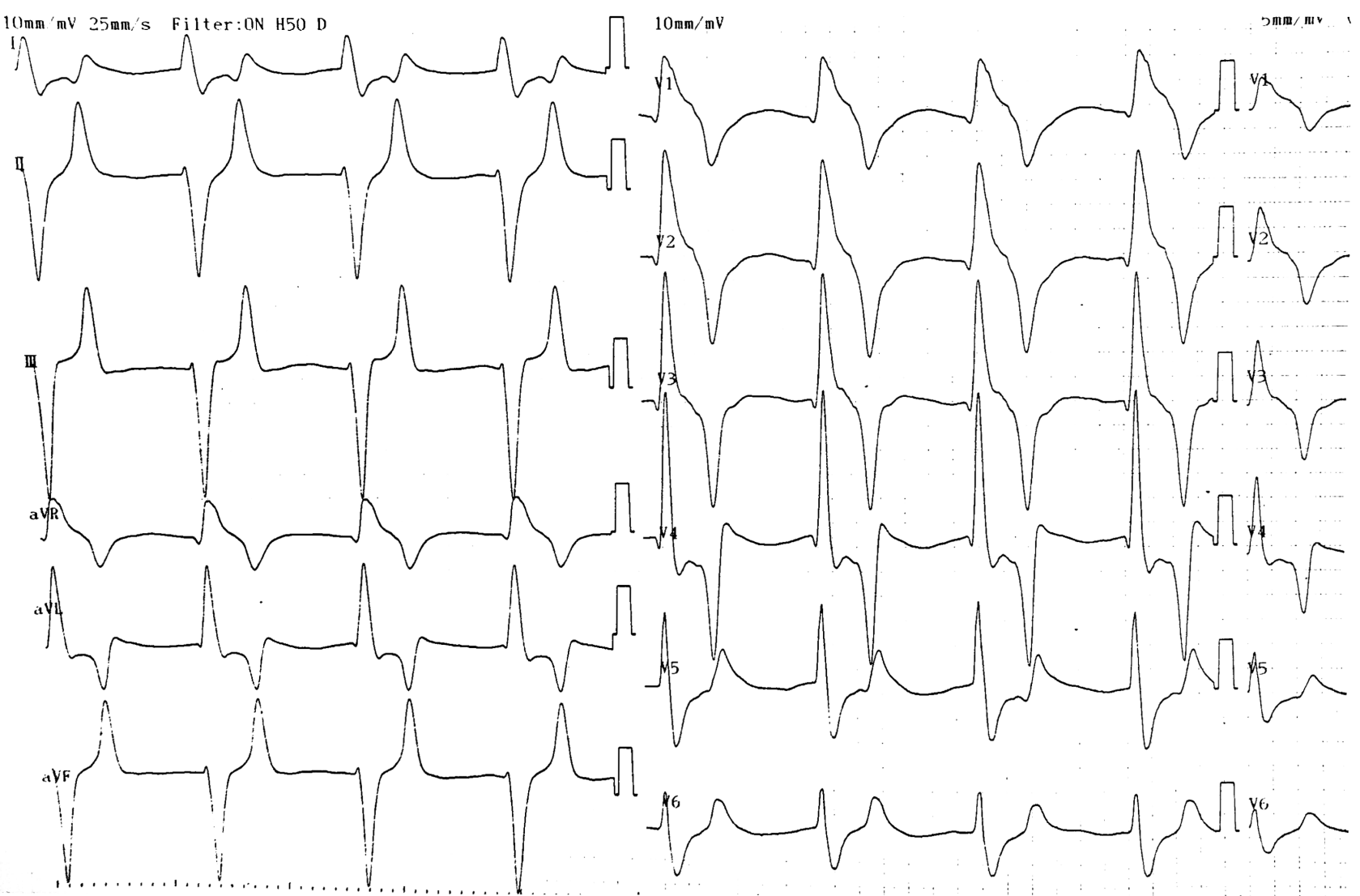

Figure 1

Electrocardiography reveals features typically associated with hyperkalemia (absent P waves, prolonged QRS interval and tented T waves).

\section{Outcome and follow-up}

Although the insulin pump tube was fully filled with insulin, the infusion line was not properly placed into the abdominal skin. The patient underwent rehabilitation, but he was unable to manage his insulin pump because of the hemiplegia and higher cortical dysfunction induced by the cerebral infarction. We therefore removed the pump and switched to intermittent insulin therapy. We performed brain CT scan twice, and there were no progression of the infarct area and brain edematous findings. On hospital day 40 , he was transferred to another hospital for further rehabilitation.

\section{Discussion}

In patients with anuria on hemodialysis, DKA is generally rare, because urinary loss of water and electrolytes does not occur and regular hemodialysis improves metabolic acidosis (2). In this patient, extreme hyperkalemia of $9.0 \mathrm{mEq} / \mathrm{L}$ with typical hyperkalemic ECG changes was observed. In general, hyperglycemia is positively correlated with the serum potassium level (3), but hyperkalemia to this extreme degree is rare. Lack of insulin action does not produce hyperglycemia alone but also causes potassium to shift from the intracellular to extracellular space by reducing $\mathrm{Na}^{+}, \mathrm{K}^{+}$-ATPase activity (4). In addition, reduced renal potassium excretion contributes to hyperkalemia in renal failure (4). This patient had a left frontal cerebral infarction resulting in right hemiplegia and higher cortical dysfunction, so we wondered if he had lost the ability to respond to hyperglycemia and handle his insulin pump properly. The present episode likely depended on absolute lack of insulin action.

In patients with diabetes who are anuric, there is a little reduction in weight and circulatory blood volume when the pathological state of DKA develops. This patient showed had some weight gain over his dry weight and cardiac enlargement on chest $\mathrm{x}$-ray. Normally, fluid infusion is essential for initial treatment of DKA (1). However, this could cause or worsen overhydration and pulmonary edema in patients with DKA who require chronic hemodialysis. Careful evaluation of body fluid volume and the serum potassium level are mandatory. If there is no volume depression, insulin treatment 
and prompt hemodialysis must be considered, but intravenous fluids should be minimized. Neurologic complications must also be assessed in patients with DKA who are on chronic hemodialysis. Hemodialysis and insulin infusion may rapidly normalize serum potassium, and plasma tonicity, largely determined by glucose and sodium, improves with the decrease in plasma glucose. Some reports have shown that rapid alteration in plasma tonicity may cause seizures or prolonged loss of consciousness because a marked change in tonicity may produce cerebral edema $(5,6)$. Hence, careful follow-up is necessary to prevent brain damage associated with reduction of tonicity by hemodialysis. In this patient, brain edema progression was not found in successive brain CT scans after hemodialysis; however, there is a possibility that rapid reduction in hyperglycemia accompanying with a marked decrease in plasma osmolality by hemodialysis and insulin infusion may mimic the prolongation of conscious disturbance. On the other hand, Daugirdas and coworkers noted that extracellular volume depletion in the central nervous system is less likely to occur in patients such as ours because hyperglycemia will not result in osmotic diuresis in an anuric patient (7). The relationship between changes in plasma tonicity and central nervous system impairment is now controversial, and the management strategy is not well established for patients with DKA who are on chronic hemodialysis. This patient's level of consciousness remained unchanged during therapy, and focal right hemiplegia was present. Thus, we performed head computed tomography to assess the focal neurologic disorder. His severe hyponatremia was concomitantly improved after the reduction in plasma glucose, and we consider that hyponatremia was secondary induced by extreme hyperglycemia (8).

In summary, we have presented a patient with type 1 diabetes on chronic hemodialysis because of anuria. He had DKA with extreme hyperkalemia, which was resolved by rapid hemodialysis and intensive insulin therapy. Although hypokalemia is common in DKA, hyperkalemia is the more likely problem in patients on hemodialysis.
Declaration of interest

The authors declare that there is no conflict of interest that could be perceived as prejudicing the impartiality of the research reported.

\section{Funding}

This research did not receive any specific grant from any funding agency in the public, commercial or not-for-profit sector.

\section{Patient consent}

Written informed consent was obtained from the patient.

\section{Author contribution statement}

$\mathrm{H}$ Yamada wrote the manuscript. $\mathrm{H}$ Yamada and S Funazaki managed this patient. M Kakei, S Ishikawa and K Hara edited the manuscript. All authors read and approved the final version of the manuscript.

\section{References}

1 Kitabchi AE, Umpierrez GE, Miles JM \& Fisher JN 2009 Hyperglycemic crises in adult patients with diabetes. Diabetes Care 32 1335-1343. (doi:10.2337/dc09-9032)

2 Lazarus JM, Denker BM \& Owen WFJ 1995 Organ system abnormalities in hemodialysis. In The Kidney, edn 5, pp 2453. Eds BM Brenner \& FCJ Rector. Philadelphia: W.B. Saunders Company.

3 Popli S, Sun Y, Tang HL, Kjellstrand CM, Tzamaloukas AH \& Ing TS 2013 Acidosis and coma in adult diabetic maintenance dialysis patients with extreme hyperglycemia. International Urology and Nephrology 45 1687-1692. (doi:10.1007/s11255013-0390-6)

4 Adrogue HJ, Lederer ED, Suki WN \& Eknoyan G 1986 Determinants of plasma potassium levels in diabetic ketoacidosis. Medicine $\mathbf{6 5}$ 163-172. (doi:10.1097/00005792-198605000-00004)

5 Tzamaloukas AH 1983 Hyperglycemia in dialysis patients. Clinical Nephrology 20323.

6 Tzamaloukas AH, Ing TS, Siamopoulos KC, Raj DS, Elisaf MS, Rohrscheib M \& Murata GH 2008 Pathophysiology and management of fluid and electrolyte disturbances in patients on chronic dialysis with severe hyperglycemia. Seminars in Dialysis 21 431-439. (doi:10.1111/j.1525-139X.2008.00464.x)

7 Daugirdas JT, Kronfol NO, Tzamaloukas AH \& Ing TS 1989 Hyperosmolar coma: cellular dehydration and the serum sodium concentration. Annals of Internal Medicine 110 855-857. (doi:10.7326/0003-4819-110-11-855)

8 Katz MA 1973 Hyperglycemia-induced hyponatremia - calculation of expected serum sodium depression. New England Journal of Medicine 289 843-844. (doi:10.1056/NEJM197310182891607)

Received in final form 18 July 2017

Accepted 20 July 2017 